\title{
LOCAL REGULARITY ANALYSIS WITH WAVELET TRANSFORM IN GEAR TOOTH FAILURE DETECTION
}

\author{
JUhani NISSILÄ \\ University of Oulu
}

\begin{abstract}
:
Diagnosing gear tooth and bearing failures in industrial power transition situations has been studied a lot but challenges still remain. This study aims to look at the problem from a more theoretical perspective. Our goal is to find out if the local regularity i.e. smoothness of the measured signal can be estimated from the vibrations of epicyclic gearboxes and if the regularity can be linked to the meshing events of the gear teeth. Previously it has been shown that the decreasing local regularity of the measured acceleration signals can reveal the inner race faults in slowly rotating bearings. The local regularity is estimated from the modulus maxima ridges of the signal's wavelet transform. In this study, the measurements come from the epicyclic gearboxes of the Kelukoski water power station (WPS). The very stable rotational speed of the WPS makes it possible to deduce that the gear mesh frequencies of the WPS and a frequency related to the rotation of the turbine blades are the most significant components in the spectra of the estimated local regularity signals
\end{abstract}

Key words: epicyclic gearbox, spectral analysis, Hölder regularity, wavelet modulus maxima, water power station

\section{INTRODUCTION}

Gear tooth and and rolling bearing faults often cause high frequency vibration which may be more evident in the higher derivatives of acceleration. In many cases the highest relative change between a faulty state and normal condition of the machine occurs when the fractional order of derivative is a real number. In [1] Kotila, Lahdelma and Ruotsalainen suggested that this may be the result of reduced regularity of the vibration signal. They also used the signal's wavelet transform to estimate its local regularity and showed that the locations of the negative Hölder regularities corresponded to where the rolling elements hit the faults on the inner race of a bearing. Here we apply the same methods to the accelerometer data from the twostage epicyclic gearbox of the WPS.

In Chapter II we present the signal processing methods. We will use the Discrete Fourier Transform (DFT) for spectral analysis, and the modulus maxima ridges of the continuous wavelet transform for local Hölder regularity estimation. This is possible due to Theorem 1, which was proved by Mallat and Hwang in 1992 [2].

Vibration measurements from the WPS are described in Chapter III and frequencies of the gearbox components are calculated. The Chapter continues with the numerical analysis of the vibration signals. Finally the obtained results are discussed in Chapter IV.

\section{SIGNAL PROCESSING THEORY}

\section{Fourier transforms}

The vibration measurements are stored as sampled sequences $x=\left(x_{0}, \ldots, x_{N-1}\right)$ of length $T=\Delta t \cdot N$,

where:

$\Delta \mathrm{t}$ is the sampling interval.
The spectrum of this sampled signal is calculated with the Discrete Fourier transform (DFT)

$$
\mathcal{F}\left\{x_{n}\right\}=X_{k}=\frac{1}{N} \sum_{n=0}^{N-1} x_{n} e^{-i 2 \pi k n / N}
$$

Its inverse transform (IDFT) is

$$
\mathcal{F}^{-1}\left\{X_{k}\right\}=x_{n}=\sum_{k=0}^{N-1} X_{k} e^{i 2 \pi k n / N}
$$

Here we have equated the inverse as $x_{n}$, because it returns the original signal at the sample points [3].

The continuous analogue of the DFT is the Fourier transform $\hat{x}$ of $x$

$$
\mathcal{F}\{x(t)\}=\hat{x}(\nu)=\int_{-\infty}^{\infty} x(t) e^{-i 2 \pi \nu t} \mathrm{~d} t
$$

Its inverse transform is defined as

$$
\mathcal{F}^{-1}\{\hat{x}(\nu)\}=\int_{-\infty}^{\infty} \hat{x}(\nu) e^{i 2 \pi \nu t} \mathrm{~d} \nu
$$

\section{Continuous wavelet transform}

Wavelet is informally defined as an oscillation with compact support. Theoretically we also allow wavelets whose amplitude decays to zero at the infinities. An admissible wavelet $\psi$ satisfies

$$
\int_{-\infty}^{\infty} \frac{|\hat{\psi}(\nu)|^{2}}{|\nu|} \mathrm{d} t<\infty
$$


The wavelet transform of $\mathrm{x}$ is then the inner product

$$
W x(s, t)=\frac{1}{s} \int_{-\infty}^{\infty} x(\tau) \psi\left(\frac{\tau-t}{s}\right) \mathrm{d} \tau
$$

Here $t$ is the point of interest in the signal and $s$ is the positive scale at which the wavelet is dilated. The admissibility condition enables the reconstruction of the original function from its wavelet transform in suitable function spaces, see for example [4]. To make this definition useful for the theory and also for the actual vibration signals, $x$ must be from the class of generalised functions called tempered distributions. Interested reader may read details about distribution theory for example from [5]. We only note that (6) is well defined if $x$ is a tempered distribution of order $m$ and if the wavelet $\psi$ is $m$ times continuously differentiable. The Fourier transform of a tempered distribution is also a tempered distribution and the inverse returns the original signal.

A wavelet has $m$ vanishing moments if

$$
\int_{-\infty}^{\infty} t^{k} \psi(t) \mathrm{d} t=0, \quad \text { for all } k=0,1, \ldots, m-1
$$

It should be noted that in practise the continuous wavelet transform is computed using only a finite sequence and summations instead of integrals. The word continuous is still used to separate the definition from the actual discrete wavelet transform where the scale $s$ is sampled at such points that the wavelets form an orthogonal set.

\section{Hölder regularity and wavelet modulus maxima}

A function $x$ is $\mu$-Hölder continuous for some $\mu \geq 0$ at the point $t_{0}$ if

$$
\left|x\left(t_{0}+h\right)-P_{m}(h)\right| \leq C|h|^{\mu}
$$

for small values of $|h|$ and $P_{m}$ is a polynomial of degree $m \leq \mu$. If $\mu$ is non-integer, it turns out that $P_{m}$ is actually the $m+1$ first coefficients of the Taylor polynomial of $x$ at $t_{0}$. If for all $t_{0}+h$ on an interval the condition (8) is satisfied, then we say that $\mu$ is the uniform Hölder exponent of $x$ on that interval.

To extend the definition to negative Hölder exponents, we utilise again distributions. Let $\mu$ be a non-integer. We say that a tempered distribution $\mathrm{x}$ of finite order is uniformly $\mu$-Hölder on the interval ] $a, b[$ if its primitive is $\mu+1$ Hölder on the same interval (primitive $=$ indefinite integral for integrable functions, see [5] for the definition for tempered distributions).

We are interested in how to detect isolated irregularities in the signal. This means that we want to locate points $t_{0}$ where $f$ is $\mu$-Hölder and also uniformly $m$-Hölder with $m>\mu$ elsewhere on the interval.

A series of local maxima or minima in the time-scale half - plane of the wavelet transform are called modulus maxima ridges. In [2] Mallat and Hwang proved that if no such ridges exist at the fine scales in a given interval, then $f$ is uniformly Hölder continuous on that interval. Thus we expect that the modulus maxima ridges will reveal the isolated irregularities in the signal and this is indeed the case. Even more is true, since the Hölder exponent can be read from the decay rate of the ridges converging to $t_{0}$ at fine scales. The next theorem is also proved in [2].
Theorem 1: Suppose that the admissable wavelet $\psi$ has compact suppport, is $\mathrm{m}$ times continuously differentiable and is the mth derivative of a smoothing function. Let $x$ be a tempered distribution and its wavelet transform well defined on ]a, b[ and let $\left.t_{0} \in\right] a, b[$. If there exists a constant $C$ and a scale $s_{0}$ such that all modulus maxima of $W x(s, t)$ belong to the cone

$$
\left|t-t_{0}\right|<C s
$$

then $x$ is uniformly $m$-Hölder in a neighbourhood of all the points $t \in] a, b\left[, t \neq t_{0}\right.$. Let $\mu<m$ be a non-integer. Then $x$ is $\mu$-Hölder at $t_{0}$ if and only if

$$
|W x(s, t)| \leq A s^{\mu}
$$

at each modulus maxima inside the cone (9).

The estimation of the local regularity is thus done in the following manner (this is a modified version of the procedure presented in [1]).

1. Compute the wavelet transform with a wavelet which has the desired number of vanishing moments.

2. Find the local minima and maxima at each computed scale.

3. Follow the minima and maxima from the smallest available scale upwards to register the modulus maxima ridges. The ridges spread approximately linearly at small scales and thus the location of maxima/minima at the next scale can be guessed by linear extrapolation.

4. Estimate also the point where the ridge ends at the fine scales by linear extrapolation in the other direction.

5. If enough maxima/minima are lined up, estimate the regularity using (10) and logarithms

$$
\log (|W x(s, t)|) \leq \log (A)+\mu \log (s)
$$

and the slope of a least squares line gives $\mu$.

6. Of all the lines converging to the same point, choose the smallest value as $\mu$.

\section{MEASUREMENTS AND REGULARITY CALCULATIONS}

Gear tooth numbers are almost always relative primes, i.e. their greatest common divisor is 1 . Thus it takes a long time for a gearbox to mesh through all of its tooth pairs. The number of revolutions it takes for all the gears to return to their original positions can be calculated with the help of some elementary number theory and these computations were done for the gearboxes of the WPS in [6]. The solutions are typically several minutes and thus not quite practical for numerical work in most cases. It is quite adequate to use signal lengths which correspond to the revolution time of the slowest rotating component in the gearbox.

\section{Water power station gearboxes}

The Kelukoski water power station has a two-stage epicyclic gearbox. The first (slower) is called gearbox 1 and the second (faster) will be called gearbox 2. Both were monitored with one WBS CM301 sensor with sampling frequency $5000 \mathrm{~Hz}$. Every 15 minutes an acceleration signal of length $7 \mathrm{~s}$ was recorded from both measurement points as WAV files. There were four continuous periods of data collection between 4.4.2013 and 22.8.2013.

Since the WPS is connected to the Finnish power grid, its output frequency is to a high precision $12,5 \mathrm{~Hz}$ (the frequency of the power grid is four times this, i.e. $50 \mathrm{~Hz}$ ). 

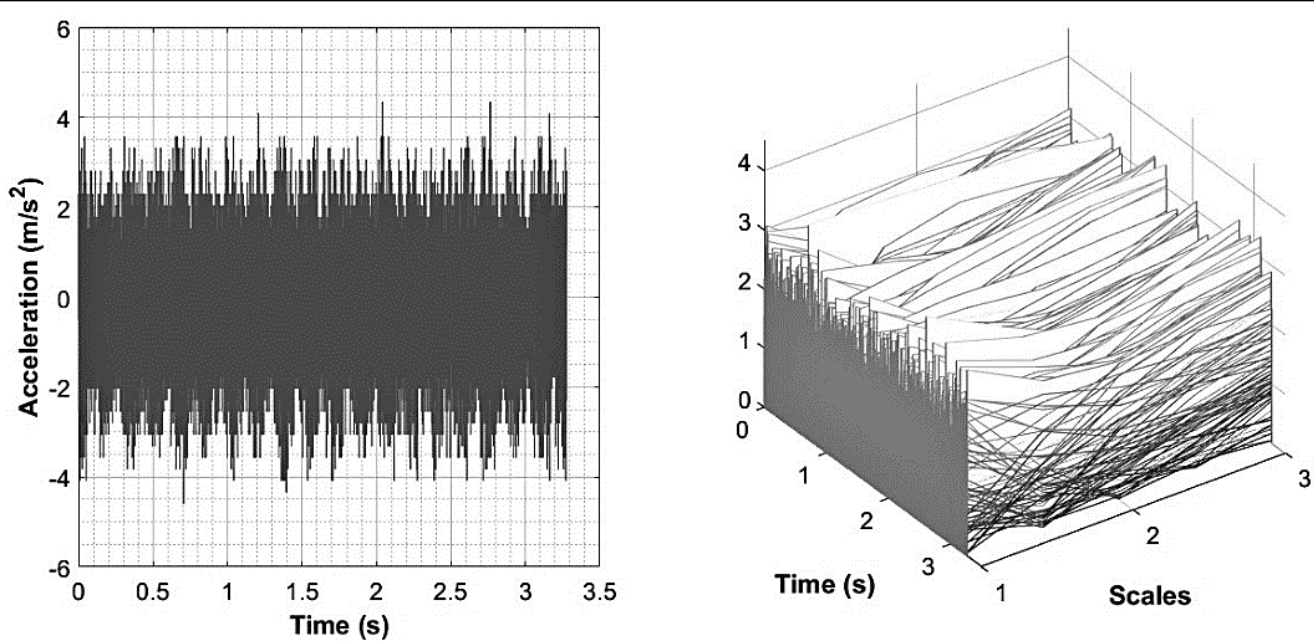

Fig. 1 Acceleration signal from the gearbox 1 recorded on 4.4 .2013 and its wavelet transform modulus
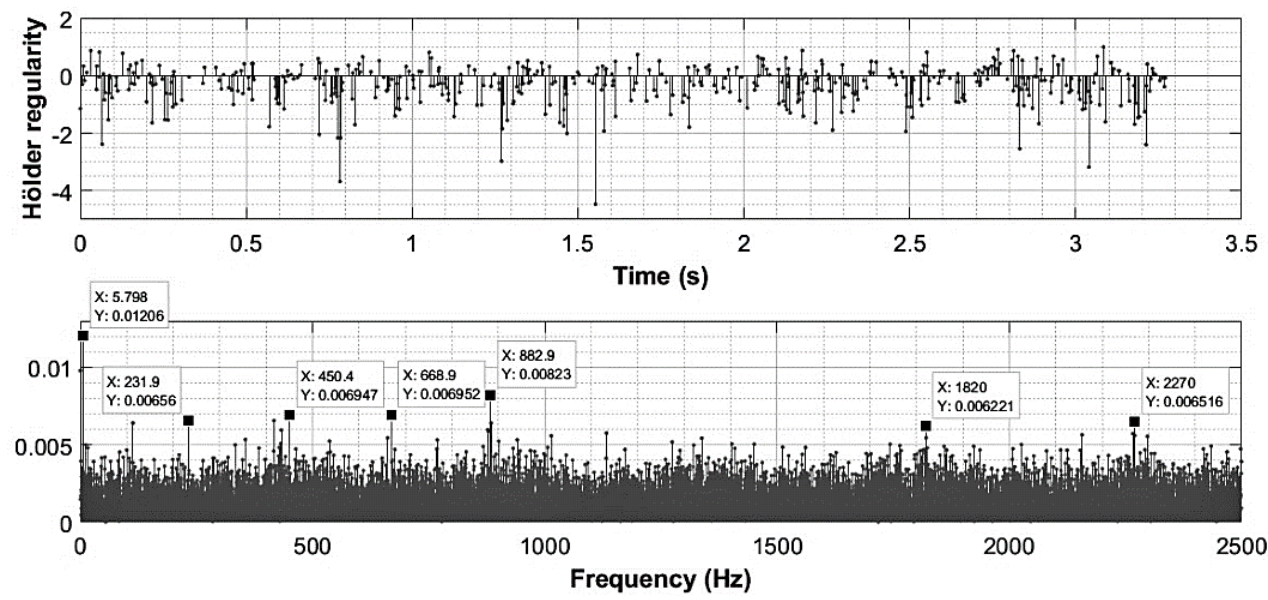

Fig. 2 Local Hölder regularity of the signal from Fig. 1 and the amplitude spectrum of the regularity signal

The characteristic frequencies of the gearboxes are now calculated backwards starting from this output. Gearbox 2 is in the star configuration, which means that it has a stationary planet carrier and six planet gears (25 teeth). Output is provided via the sun gear ( 36 teeth), $v_{\text {sunW P S2 }}=12,5$ $\mathrm{Hz}$, and input from the gearbox 1 via the ring gear (86 teeth). The gear teeth are double helical and the gearboxes have plain bearings. The formulas for the frequencies are very simple in the star configuration [7]

$$
\begin{gathered}
\nu_{\text {ringW PS } 2}=-\frac{36}{86} \nu_{\text {sunW PS } 2} \approx-5.23 \mathrm{~Hz} \\
\nu_{\text {planetsW PS } 2}=\frac{86}{25} \nu_{\text {ringW } P S 2}=-18.00 \mathrm{~Hz}
\end{gathered}
$$

and the mesh frequency $v_{\text {meshWPS2 }}=36 \cdot v_{\text {sunWPS2 }}=450.00$ $\mathrm{Hz}$.

Gearbox 1 is in planetary configuration, meaning that it has a stationary ring gear. The sun gear is the output and has 31 teeth and the planetary gears have 25 teeth. The ring gear has 81 teeth. We have $v_{\text {sunWPS1 }}=v_{\text {ringWPS2 }}$ (this middle part of the two gearboxes is a floating installation) and the frequencies are

$$
\begin{aligned}
\nu_{\text {carrierW } P S 1}=\frac{31}{31+81} \nu_{\text {sunW } P S 1} & =\frac{31}{112} \nu_{\text {sunW } P S 1} \\
& \approx-1.45 \mathrm{~Hz}
\end{aligned}
$$

$$
\begin{aligned}
\nu_{\text {planetsW PS } 1} & =-\frac{81-25}{25} \nu_{\text {carrierW } P S 1} \\
& =-\frac{56}{25} \nu_{\text {carrierW } P S 1} \approx 3.24 \mathrm{~Hz}
\end{aligned}
$$

and finally the frequency

$$
\nu_{\text {meshWPS } 1}=81 *\left|\nu_{\text {carrierWPS } 1}\right| \approx 117.31 \mathrm{~Hz}
$$

Measurements from the WPS have previously been studied in $[6,8,9]$. Unfortunately, before the previous breakdown of gearbox 1 several years ago, it had a slightly different set of gears with 35 teeth in the sun gear, 27 in the planetary gears and 91 in the ring gear. These give the same ratio for the output of the gearbox, but the frequency of the planetary gears was $3.45 \mathrm{~Hz}$ and most importantly the mesh frequency was $131.80 \mathrm{~Hz}$. Due to some problems with communication these wrong gear numbers were used in the publications $[8,9]$. Obviously the lack of frequency $131.80 \mathrm{~Hz}$ and the appearance of frequency $117.3 \mathrm{~Hz}$ in the spectra was a puzzle before this error was spotted.

Local regularity analysis of measurements from the WPS

One measurement from both gearboxes was analysed from the beginning and end of the measurement period. The output power of the WPS was about 7.6 MW during those measurements. The signals were shortened to $2^{14}=16384$ samples, which gives a length of $16384 /(5000 \mathrm{~Hz})$ $=3.2768 \mathrm{~s}$. Next the continuous wavelet transform (6) was 
computed at scales 1, 1.5, 2, 2.5 and 3 using the Mexican hat wavelet. It is the negative normalised second derivative of a Gaussian function and has two vanishing moments. Obviously it does not have exactly compact support but in practise this has little effect on the results. Computations were carried out with MATLAB. In the algorithm, a ridge was confirmed if a maxima was found from all the computed scales and they were approximately linearly spaced (a deviation of one discrete sample in both directions was allowed here in the search of a maxima from the current scale).

Fig. 1 and 3 show the measured vibration signals from gearbox 1 and the modulus of their wavelet transforms. The measurements were recorded on 4.4.2013 and 22.8.2013 respectively. The resolution of the accelerometer is clearly visible in these low vibration levels. There seem to be both ascending and descending ridges with increasing scale, which implies both positive and negative Hölder regularities in the signals. Fig. 2 and 4 show the estimated local regularities from these signals and also their amplitude spectra. We see that indeed both signals oscillate between positive and negative regularities and that the maximum regularity value we can analyse with the Mexican hat wavelet (which is 2 according to Theorem 1 ) is enough for these signals. For the calculation of the DFT of the regularity signal, the missing values are assumed to be zero. This is convenient, since the DFT needs an equally spaced vector. It must be mentioned though, that the signal is possibly infinitely smooth at these points and thus inserting Hölder coefficient 0 to them is a crude simplification to make the DFT easily computable.

It seems that the vibrations of gearbox 1 have not changed noticeably during this short measurement period (although in [6] a slight increase in the norms calculated from the second derivatives of these acceleration signals was observed), so we will discuss the two measurements simultaneously. It is interesting that although the spectra of both local regularity signals contains an almost uniform base across frequencies (that is the spectral content of white noise), there are also spikes at certain frequencies. Most of these are near the multiples of the mesh frequencies of the gearboxes, such as $2 * v_{\text {meshwPS1 }} \approx 234 \mathrm{~Hz}, 4 * v_{\text {meshWPS1 }}$ $\approx 469 \mathrm{~Hz}$ and $2 * v_{\text {meshwPS2 }}=900 \mathrm{~Hz}, 4 * v_{\text {meshwPS2 }}=1800 \mathrm{~Hz}$, $5 * v_{\text {meshWP } 22}=2250 \mathrm{~Hz}$. The higher speed and bigger vibration levels explain why the multiples of the mesh frequency of gearbox 2 is also visible here. The sidebands which are $18 \mathrm{~Hz}$ apart from $\mathrm{v}_{\text {meshwPs2 }}$ and its multiples are sometimes

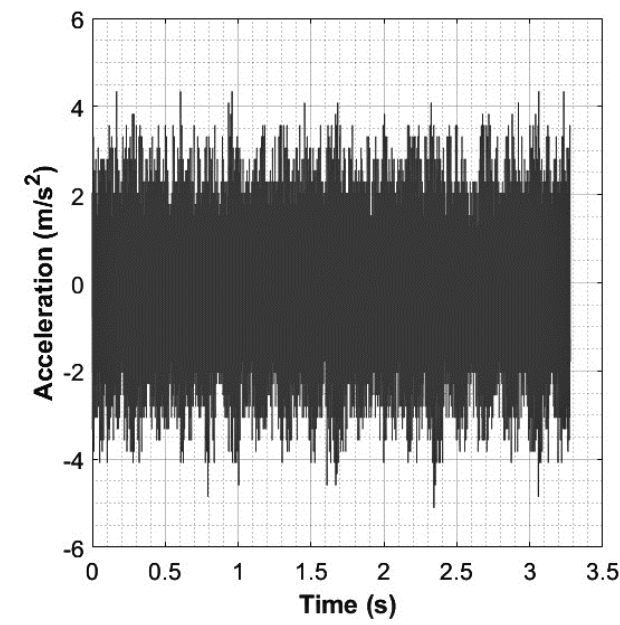

even more noticeable than the center frequencies. Thus the frequency $\approx 469 \mathrm{~Hz}$ mentioned above may actually be such a sideband also. These are of course caused by the rotation of the planetary gears of gearbox 2 .

The biggest change with time is the increase in the frequencies near $2250 \mathrm{~Hz}$. Interestingly, this was also the only major change observed in the spectra which were calculated directly from the acceleration signals from the same measurement period in [6]. It should also be noted that since these frequency spikes are so discrete, this change is not so huge if one notices that the closest neighbouring frequencies of $2270 \mathrm{~Hz}$ in Fig. 2 are also quite noticeable.

There is also one very low frequency spike at about $5.8 \mathrm{~Hz}$. This oscillation with period $1 / 5.8 \mathrm{~Hz} \approx 0.17 \mathrm{~s}$ is visible in the time domain signals as well. This frequency could be $4 * v_{\text {carrierWPS1 }}$ or even maybe $\mathrm{V}_{\text {ringWPS2 }}$ due to inaccuracies in the numerical work and the frequency resolution. The turbine has four blades and is connected to the carrier of the gearbox 1 , which suggests that $4 * v_{\text {carrierwPS1 }}$ is probably the correct answer. Thus we could deduce that that local irregularities in the acceleration measurements from the gearbox 1 are caused by the rotating turbine blades and the gear meshes from both gearboxes.

Fig. 5 and 7 show the measured vibration signals and their wavelet transform modulus at the beginning and at the end of the measurement period from the gearbox 2 . Now almost all of the wavelet maxima modulus ridges increase towards fine scales and we thus expect a lot of negative regularities. Fig. 6 shows the estimated local Hölder regularity of the signal from the beginning of the measurement period and the amplitude spectrum of this signal. We see that the signal has mainly Hölder exponents between 0 and -3 . The amplitude spectrum is dominated by $v_{\text {meshwPS2 }}$ $=450 \mathrm{~Hz}$ and its multiples. The higher multiples also have sidebands which are about $50 \mathrm{~Hz}$ above them. Fig. 8 shows similar results from the end of the measurement period, although now we also see more clearly the sidebands $18 \mathrm{~Hz}$ apart from $v_{\text {meshwPs2 }}$ and its multiples. The higher speed gearbox 2 thus shows quite clearly that the local irregularities of its measured acceleration signals are mostly caused by the meshing events of its gears. Comparing the spectra from Fig. 6 and 8 we also see that $v_{\text {meshwPS2 }}$ and its multiples have decreased. This is also visible in the fewer amount of irregularities in the time domain signal in Fig. 8. It may just be that the algorithm failed to recognise as many ridges in the later signal or perhaps the vibration has actually become smoother with time.

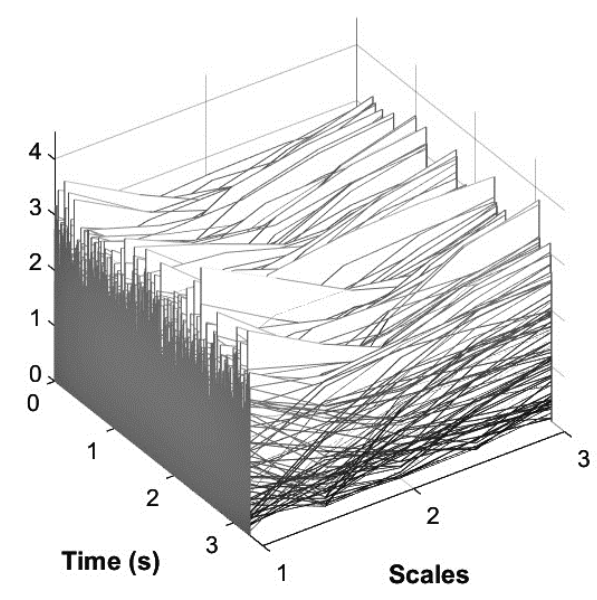

Fig. 3 Acceleration signal from the gearbox 1 recorded on 22.8.2013 and its wavelet transform modulus 

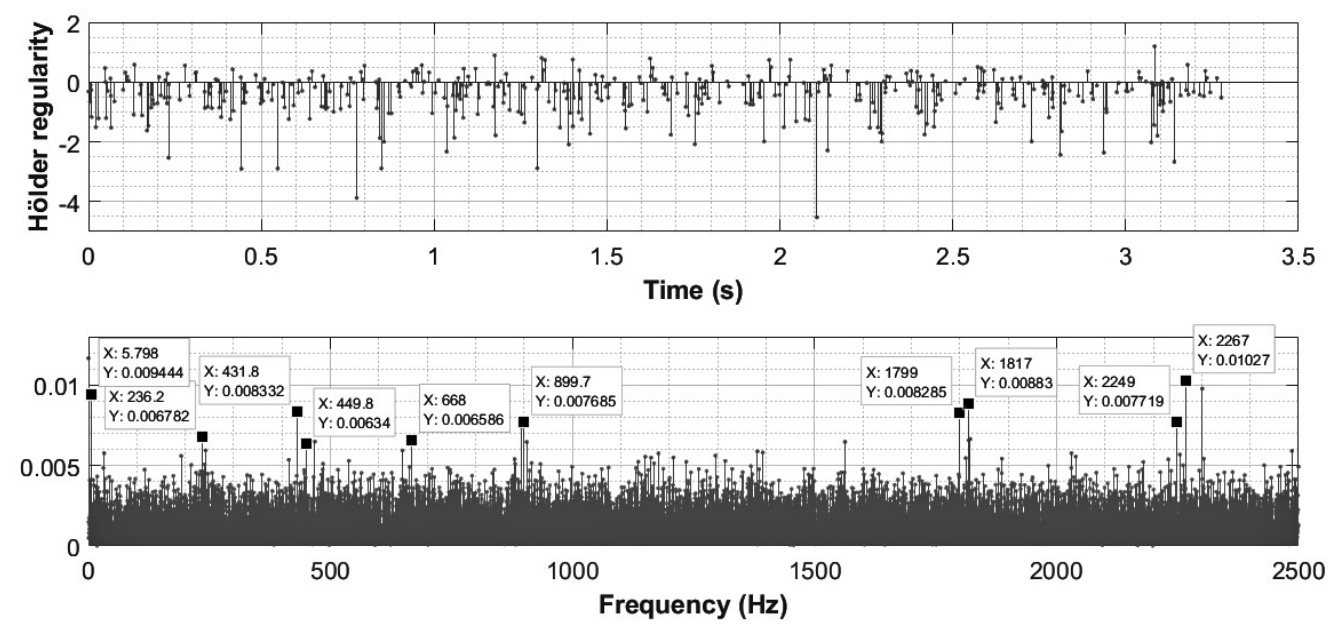

Fig. 4 Local Hölder regularity of the signal from Fig. 3 and the amplitude spectrum of the regularity signal
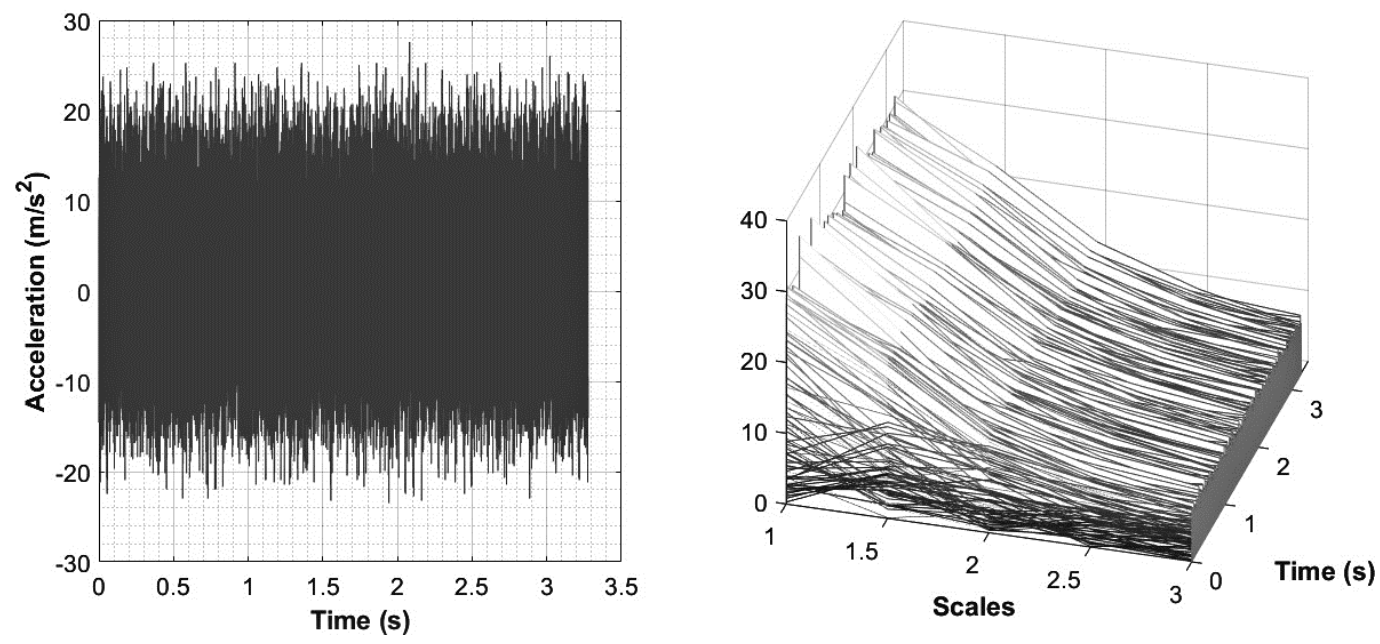

Fig. 5 Acceleration signal from the gearbox 2 recorded on 4.4.2013 and its wavelet transform modulus
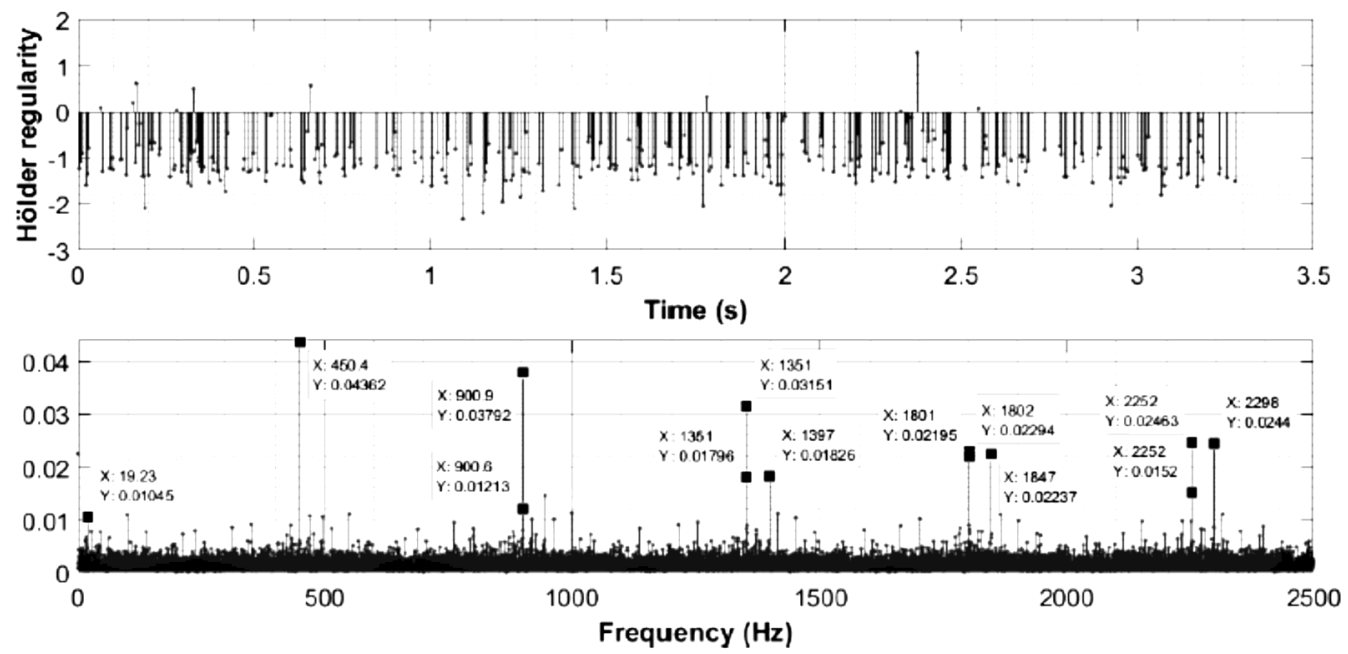

Fig. 6 Local Hölder regularity of the signal from Fig. 5 and the amplitude spectrum of the regularity signal 

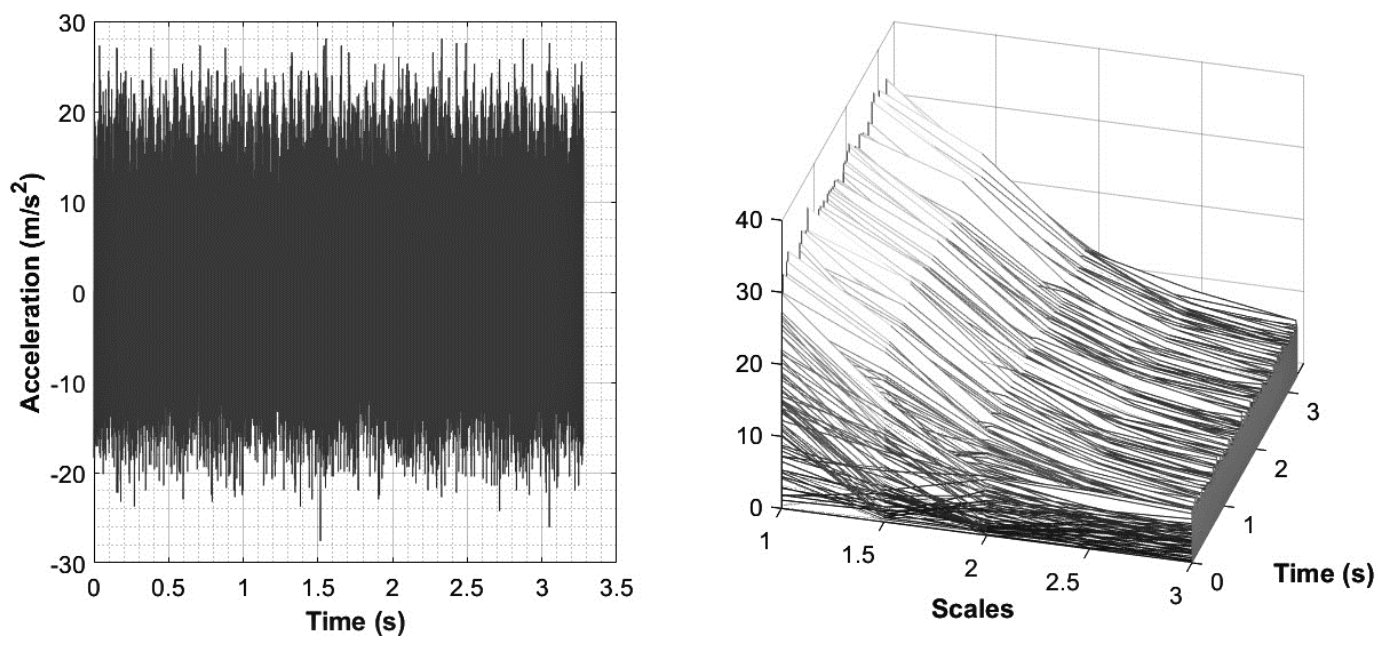

Fig. 7 Acceleration signal from the gearbox 2 recorded on 22.8 .2013 and its wavelet transform modulus
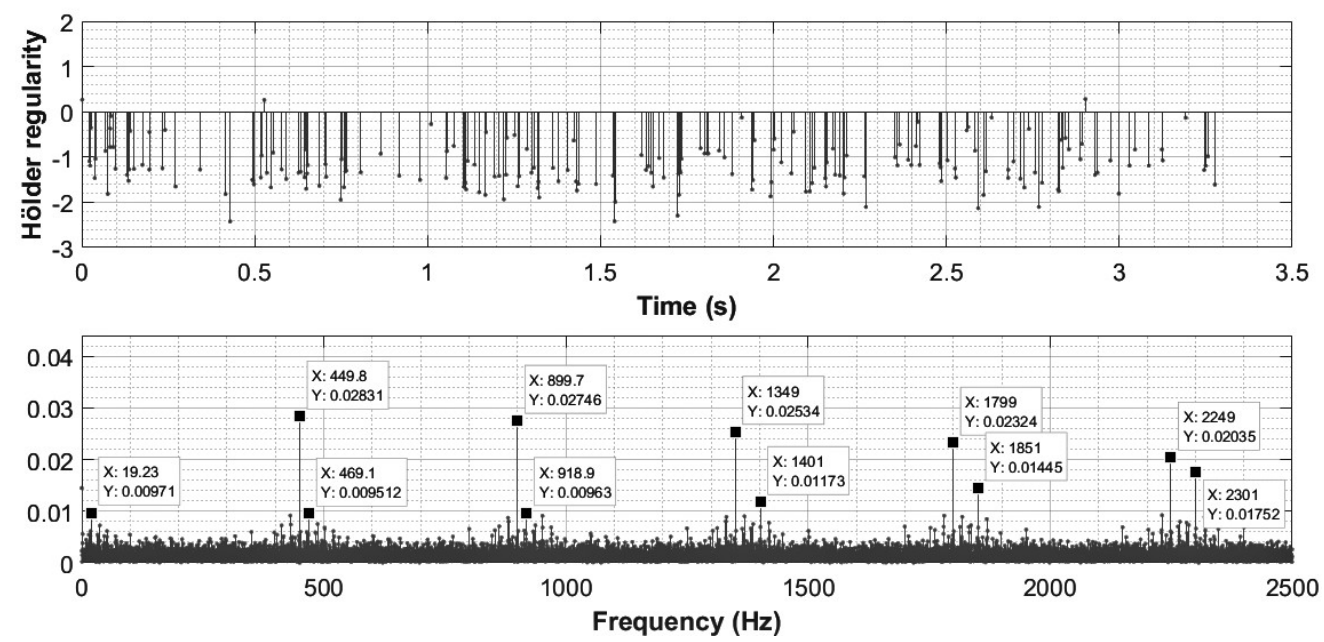

Fig. 8 Local Hölder regularity of the signal from Fig. 7 and the amplitude spectrum of the regularity signal

\section{CONCLUSIONS}

For machines which rotate very precisely at a constant speed it is possible to calculate the DFT of the estimated local regularity signal and it may contain sharp spikes related to the rotating components of the machine. In this paper we have shown this using measurements from the twostage epicyclic gearbox of a water power station.

Minor changes in these spectral components were also observed during the almost four-month long measurement period. This could be an indication of wear on the gears, but this is far from certain. The measurement period was very short considering that we can not rule out for example seasonal effects which may cause different levels of stress on the gearbox. The true condition of the gears is also unknown. The estimation of the irregularities in the signals is also a nontrivial pattern recognition task and thus the results obviously contain errors. Averaged results from many signals could provide more reliable results. Spectral content directly from the acceleration signals from the same measurement period was analysed in [6] and very minor changes were observed in those as well.

The newest version R2016B of MATLAB introduced the built-in function wtmm for locating the wavelet modulus maxima ridges and estimating the local regularity. Unfortunately, it is not yet thoroughly documented and it seems that it searches the ridges starting from the big scales and then occasionally fails quite badly in locating the point where the ridge should converge. For this reason the author wrote his own code for the task.

Longer studies from industrial sites as well as test bench studies will be done to learn more about the connection between the regularity of vibration and the machine's health. If the machine does not rotate exactly at a constant speed, then it is unlikely that the DFT could catch any discrete spectral components from the regularity signal. In these cases the signal should at first be order-tracked. This can be done relatively easily if a tacho signal, which reveals the rotational speed of the shaft, is available. Another option could be to utilise the cyclostationarity of such a signal with the methods of cyclic spectral analysis.

The measurements at the WPS were part of the "Integrated condition-based control and maintenance (ICBCOM)" project. Author wishes to thank Otto A. Malm Foundation for their support on his doctoral studies.

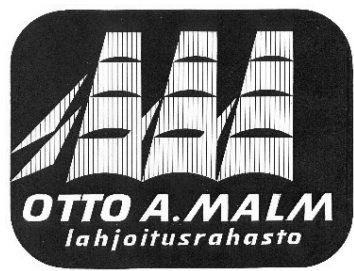




\section{REFERENCES}

[1] V. Kotila, S. Lahdelma and K. Ruotsalainen, "Wavelet -based Hölder regularity analysis in condition monitoring", in Integral Methods in Science and Engineering. Computational methods, vol. 2, M.E. Pérez, Ed. Boston: Birkhäuser, 2010, pp. 233-242.

[2] S. Mallat and W.L. Hwang, "Singularity detection and processing with wavelets", IEEE Transactions on Information Theory, vol. 38, no. 2, pp. 617-643, 1992.

[3] W. Briggs and V.E. Henson, The DFT An Owner's Manual for the Discrete Fourier Transform. Philadelphia (PA): Society for Industrial and Applied Mathematics, 1995.

[4] M.A. Pinsky, Introduction to Fourier analysis and Wavelets. Pacific Grove: Brooks/Cole, 2002.

[5] A.H. Zemanian, Distribution Theory and Transform Analysis: An Introduction to Generalized Functions, with Applications. New York: Dover Publications, 1987.

[6] J. Nissilä and E. Juuso, "Extracting vibration severity time histories from epicyclic gearboxes", in Proc. The $9^{\text {th }}$ Eurosim Congress on Modelling and Simulation, Oulu, Finland, 2016, pp. 385-391.

\section{Juhani Nissilä MSc}

Faculty of Information Technology and Electrical Engineering Applied and computational mathematics,

University of Oulu

Pentti Kaiteran katu 1, 90014 Oulu, FINLAND

e-mail: juhani.nissila@oulu.fi
[7] C.M. Vicuña, "Contributions to the analysis of vibrations and acoustic emissions for the condition monitoring of epicyclic gearboxes", Ph.D. dissertation, Aachener Schriften zur Rohstoff- und Entsorgungstechnik des Instituts für Maschinentechnik der Rohstoffindustrie, Institut für Maschinentechnik der Rohstoffindustrie, 2010.

[8] J. Immonen, S. Lahdelma and E. Juuso, "Condition monitoring of an epicyclic gearbox at a water power station", in Proc. The 53rd Scandinavian Conference on Simulation and Modelling, Reykjavik, Iceland, 2012, pp. 99-105.

[9] R.P. Nikula, K. Leiviskä and K. Karioja, "Epicyclic gearbox monitoring in a hydroelectric power plant with varying load", in Proc. $12^{\text {th }}$ International Conference on Condition Monitoring and Machinery Failure Prevention Technologies, Oxford, UK, 2015. 